\title{
The role of research in cave mining
}

\author{
SD McKinnon Queen's University, Canada
}

GA Ferguson Seltrust Associates Limited, UK

\begin{abstract}
There are many reasons to carry out research in mining operations and its associated technical disciplines, such as solving fundamental problems and adapting new technologies to the mining environment. Carrying out research is not the primary business of mining companies, although it is vital to the long-term health of the industry. In this paper, the differences between fundamental research, innovation and consulting are described, as is how to identify where on the research and development (R\&D) spectrum a research project should be positioned. Further, the role of different organisations in conducting R\&D work is discussed. Planning and managing research projects and programs can have a significant influence on the cost and success of projects even if they are outsourced to specialist practitioners, which is a common practice in research work today. An example of how the planning and positioning of R\&D projects can be assisted by classification methods is given. Finally, some research areas that are of particular relevance to cave mining are suggested.
\end{abstract}

Keywords: $R \& D$, innovation, cave mining problems, mining-induced seismicity, preconditioning

\section{Introduction}

Research investment varies significantly across different sectors of the economy, but in general, mining is not known as a research-intensive industry. Based on benchmarking of R\&D expenditures in other industries, mining companies should contemplate increasing their R\&D investments to $2-4 \%$ of revenues (Leach 2014). It would also be very beneficial for R\&D investment by key suppliers in joint projects to increase spending from the current $1 \%$ to $3-4 \%$. These levels of spending are consistent with approaches in the oil and gas industry. The reasons for this low expenditure in R\&D are numerous and complex, but significant factors include the long-term capital-intensive nature of mining projects, coupled with the strongly cyclical demand for commodities. Under such circumstances, investment spending must be conservative, minimisation of risk is a priority, and the reliability of well-established practices is favoured. The role of research, and the development of its practitioners, varies widely across the sector. In its most fundamental form, the results of research may only be realised in the timeframe of a decade or more, which is far from the concern of most managers. At the other end of the R\&D spectrum, the task is generally to apply the results from early-stage research to mining problems. This 'innovation' form of research operates on shorter timescales, typically requires conversion from prototype to industry-ready products, and implementation may require training and changes in workflow. Management of these diverse forms of research over a wide range of timescales, with their different skill set and resource requirements, is quite different from management of mining operations, and therefore represents a challenge for its value to be realised.

Drivers of research may involve factors either internal or external to mining operations. Problems arising from internal factors may be managed by adapting existing techniques of practice, but ultimately new approaches may be needed, requiring research. A simple example of this progression is the nature of rock mass behaviour as depth increases. At shallow to intermediate depth, fracturing may be limited and aseismic. However, below some critical depth, the rock mass response may become seismically active, requiring a completely different approach to both mine layout and sequencing for large-scale stress management, including new types of ground support to manage the dynamic loading of fractured rock around excavations. Significant changes in behaviour such as this may occur rapidly, unexpectedly, and exhibit a response beyond the experience base of the mine. External drivers of research involve factors that are typically outside of the 
control of the mining industry, such as macroeconomic and socioeconomic changes, availability of new technologies (e.g. automation) or other so-called disruptors. Challenges arising from such external factors can be very difficult to manage and respond to since they may require skills that do not typically exist within the industry, i.e. community relations, ecological sciences, or geopolitical risk management.

As with any activity, research has special requirements for it to produce value. Research can be easily dismissed as costly, requiring too much time to complete, or be labelled as impractical. However, correctly managed, it can be highly effective. In this paper, commentary is provided on conducting research in the mining industry that can increase its successful planning and execution. Some specific applications related to cave mining are presented. To facilitate the discussion, several themes that affect decisions on its management have been identified.

\section{Why mining companies need to do research}

Carrying out research is not the primary business of mining companies, but it is vital to the long-term health of the industry. There are many reasons to carry out research in mining and its associated technical disciplines, but they principally involve the following:

- Improving safety, efficiencies, economics.

- Solving technical problems arising in operations in cases where existing knowledge or techniques are inadequate.

- Taking advantage of new technologies.

- Advancing the technical skills of personnel.

Research involves a wide range of timescales, assumes various forms, and may be done by a wide range of people with similarly diverse skills; i.e. research is not simply a single catch-all task, and understanding these various aspects of research and their interplay is important in planning, managing, and implementing research.

\section{Types of research and research providers}

Solving problems is the function of all engineering, but depending on the nature of the problem, the approach taken can be quite different, and the skills, timing, risks, and funding also vary. Since research is not the core business of mining, there is often no clear definition or even understanding of these divisions, and consequently, there can be misclassification and inefficient assignment of tasks. Even within the traditional research community (university researchers) there is debate over the boundaries of what should be classified as research and who should do it, and there is clearly overlap between the different classes of research and research organisations. With this in mind, some key factors to consider are outlined herein.

\subsection{Basic and applied research}

There are many definitions of research, but the most common characteristic is that it involves the creation of new knowledge or theories, typically developed to improve understanding. It may also involve extension of existing knowledge that was not previously considered or required. Research may be of a fundamental nature (basic research), or directed towards the solution of a specific problem (applied research). The knowledge gained through research enables solutions to practical problems to be more reliably developed. Research work underpins and is a requirement for the success of all upstream developments, including innovation. Since its purpose is to develop new knowledge, there will be no established problem-solving methodology to follow, so results, and timelines, are hard to predict. Research is therefore a high-risk activity. However, there is generally higher risk associated with not doing research if it delays development of critical knowledge. 
The path to creating new knowledge is never clear and may involve closing unfruitful avenues of investigation. There is generally greater uncertainty in the outcome of basic research compared to applied research, and the path forward may be less linear. Deviations in the research plan in response to incremental results are therefore a natural part of basic research and should not be classified as failures. Monitoring the progress of such activities should also be selected to be appropriate to the time horizons involved, e.g. quarterly or bi-annual reporting is not typically relevant.

The long-term nature of basic, and to some extent applied research, is best sustained by generally moderate levels of funding as opposed to periodic large sums. Related to this long-term maintenance is the parallel requirement to develop the skills of personnel to conduct that form of work. Since the pathway for progress cannot be known in advance when pursuing new knowledge, more specialised and deeper knowledge of the existing state-of-the-art, and familiarity with the literature of related research results and research methodology is required. This skill set differs significantly from that required to succeed in a production environment, although its development follows a similarly managed career path. Maintenance and development of these skills therefore needs to be nurtured over the long-term either by a commitment to maintaining a research base in-house, or by developing and maintaining a long-term partnership with external providers such as research organisations or universities. In either model, for maximum effectiveness in creating value for the mining industry, interaction between researchers and industry, and access to mines, is a vital component of this long-term skill development and industry problem-solving capacity.

As a cautionary note to the long-term maintenance of the research base, there have been instances when urgent needs have driven large sums of funding into research projects, with the intention of accelerating the development of results that can be applied in mines. Without the underpinning of basic research, the pipeline to solving practical problems has limited capacity, unless the basic research is already available from other disciplines. Furthermore, the long-term nature of research groups and the personnel involved make expansion in the short-term a difficult proposition. Owing to the long-term development and scarcity of basic research personnel, the capacity to absorb increased funding is quickly saturated, and can lead to disillusionment about the capability of research to solve the problem. The fault in these cases is not that of the research programs or personnel, but lack of recognition of the timescales between basic research and innovation.

\subsection{Innovation}

The most important aspect of the research and development (R\&D) spectrum for mining companies is in the development, or innovation end, since there are many other disciplines related to mining that have significantly larger supply chains of R\&D that can be tapped into. For example, earth science research feeds exploration methods and equipment; computer science, electrical control systems and mechanical engineering are giving rise to artificial intelligence (Al), machine learning and robotics, which feeds developments in autonomous equipment.

Innovation entails developing new applications from existing knowledge, whereas basic research is about developing new knowledge. This is an important distinction as innovation and basic research require different timeframes and methodologies to complete the tasks in hand.

Technological changes over the lifetime of large-scale mining projects can be dramatic. In order to take advantage of these developments, it must be adapted for use in the mining industry. This is the very definition of innovation; i.e. the fundamental research responsible for the technological developments has already been done, but new applications for that technology remain to be adapted to the mining industry. The availability of this wealth of technology developed primarily outside of mining creates an incentive to put greater emphasis on innovation than on basic research within mining. However, not all solutions to mining problems can be solved this way, and fundamental mining-specific research is still required. 


\subsection{Empirical research}

"For every complex problem there is an answer that is clear, simple, and wrong." (Mencken 1917)

Very often, pressure of production and the urgency of problems do not permit long-term investigation, and quick solutions are required. If sufficient cases or experience are available to draw from, it is possible to develop empirical relationships. These do not require the underlying control mechanisms to be understood, but when developing such relationships, it is critical to incorporate key controlling variables. The main lesson is that to be useful, it is not necessary to fully understand the problem or behaviour. An important limitation is that the use of such relationships outside of their calibrated experience base may lead to unexpected and unreliable results.

\subsection{Consulting companies and research}

Consultants do not generally undertake research, although they could, albeit at a high cost. Consultants do, however, specialise in the application of known analysis techniques and methodologies, which is generally best suited to solving short-term operational problems and long-term planning within the experience base of conventional practice. Through their work on many projects for a variety of companies, consultants may be considered a repository of industry knowledge and practice, made available for hire (Hustrulid 2006). Such circumstance does not preclude the application of specialised analysis techniques in new ways, which is classified more appropriately as innovation, as opposed to fundamental research.

\section{$4 \quad$ Research organisations}

Various organisations exist today worldwide involved in mining research and funded from a variety of sources and stakeholders; e.g. universities, in-house technical services, and contract research organisations.

\subsection{Universities}

Universities carry out research, but are historically set up for fundamental research rather than for operationsbased research. Accordingly, the current model of university research is not well suited to solving complex industrial problems in relatively short timeframes, although the model does provide a very good environment in which experienced professionals can incubate solutions to complex problems. A typical timeframe to complete a PhD is, at minimum, four to five years, so complex problems cannot be solved quickly. Within such a long time horizon environment, it is important to understand the potential for a lack of results. Although practice varies round the world, most postgraduate programs are set up for young professionals, often continuing their undergraduate studies with no professional experience. Postgraduate degrees have an important training element in the methods of research, and the development of technical skills remains an invaluable contribution of university research. However, as salary structures indicate, this added training is typically not recognised by the industry, and the time commitments required make it difficult for working professionals to take the two to five years of time required to complete graduate degrees.

Consequently, the expertise available to work on university research projects, while often of a high technical calibre, often lacks the experience component that practicing engineers can bring to a project. Experienced supervisors can compensate somewhat for this, but that is not the principal role of academic supervisors.

University-based research offers a number of advantages for non-critical path projects. Their low-cost structure and frequent ability to leverage government funding programs makes them ideal to undertake higher-risk, longer-term investigations of difficult problems, and the specialist training aspect should not be overlooked. These activities are best sustained by lower levels of funding as opposed to large fluctuations, which are difficult to accommodate. 
Engagement of professors in industry-based research is important so that they are free from the 'ivory tower' syndrome. This is a long-term relationship that needs to be nurtured by the mining industry (e.g. Minerals Council of Australia 1998), but in many countries it is not current practice.

\subsection{The ideal research organisation}

It is useful to consider the characteristics of an ideal mining R\&D organisation, with a number of essential elements:

- Carries out a broad spectrum of investigation, spanning from fundamental research to refinement of applications for implementation into operations.

- Has a range of practitioners to carry out a broad research program.

- Needs a critical mass of researchers and resources to be effective.

- Is well connected to mines, so projects are needs-driven.

- The role and importance of research needs to be understood by the mining industry so that research related work can be conducted and integrated in operating mines with shared goals. Performance indicators at operating mines should allow for work and resources required for non-production related tasks of research.

In short, the management and structure of research is not that much different from other organisations, with the exception that it can be very difficult to measure the financial benefit of its products, and in fact, experience shows that it is often considered as a cost, not a benefit. There have at times been some highly effective research organisations in the mining industry. In the past, the Chamber of Mines of South Africa, created and owned by the gold mining industry, is one example. Today, research is highly fragmented and faces challenges because of that. Critical mass, limited funding, fragmentation of direction, and competition between researchers all damage productivity. It is not difficult to see that the research culture in mining lags that of many other industries. Can this situation be remedied?

\section{$5 \quad$ General aspects of research}

Project management considers the three metrics of cost, time and scope. Research can be thought of in similar terms, but we add another important factor - risk.

\subsection{Research is subject to uncertainty}

The uncertain path to solutions makes research an inherently risky business. Fundamental research has the greatest uncertainty, since it is the pursuit of new knowledge. Development and innovation research are built on the foundation of fundamental research, thus reducing risk. The variable nature of risk needs to be correctly identified as part of the management process. The authors are aware of only one research funding agency that required identification of the risk factors to research outcomes as part of the proposal process. Most agencies are risk averse and require specification of milestones, which the authors believe to be counterproductive to encouraging creativity in fundamental research. Strict reinforcement of deliverables will force researchers to plan conservatively. Correct classification and identification of risk is therefore an integral component of managing research.

\subsection{Time scales in research}

The majority of technical problems arising in mining operations are adequately addressed by internal specialists or externally by consultants. However, certain types of problems cannot be solved using existing techniques, even by experienced specialists. In these cases, new solutions are required, involving research. Whereas the timeframe for solving problems using existing techniques may be weeks or months, the timescales for research involving discovery of new knowledge can be years. Therefore, research is a 
long-term endeavour that cannot be applied to solve short-term needs no matter how critical to the operation. For this reason, it is easy to make the decision not to do any research, since it often has no short-term economic benefit. Similarly, it is important that chronic or systemic problems of the industry be identified, and a strategy formulated to solve them. Long-term research typically does not require major funding, but underfunding or ignoring it ensures that the foundations for future innovations will be absent.

\subsection{Conducting research in mining operations}

Amos Tversky wrote: "The secret to doing good research is always to be a little under-employed. You waste years by not being able to waste hours." (Lewis 2016)

How best to go about mining research in operations requires consideration of a number of dynamics:

- The mines are the laboratories of mining research, and the driver of the research program, so there has to be tight integration of research within operations.

- It is ideal if researchers are attached to and have good access to mines.

- Dedicated research personnel working within a project team is a sound approach, as opposed to research being tasked to engineering staff with operational roles. Such an arrangement generally leads to failure, since short-term demands of operations will always take precedence (as they need to).

\subsection{Implementation of research results into practice}

Of course, once a project is reaching fruition, it needs to be formally taken up by the mine operational staff and fully implemented. A well-structured research program will have this step fully integrated through the development stage, working together with the mine staff and workforce to demonstrate the benefits of the research project. Without integration, it is quite possible for solutions to sit on the shelf, ignored and forgotten.

Change management will ordinarily be required to facilitate the adoption of the solution, given the likelihood that operations will have to change to adopt the results of the research work.

\section{$6 \quad$ Identifying research priorities}

Considering the diversity of research topics and where they fall on the R\&D spectrum, setting priorities and allocating appropriate resources can be a challenging task. Two effective methods in this regard are risk assessment and the methodology of classification systems.

\subsection{Risk assessment of problems}

There are many methods for assessing risk, several of which are commonly used in the mining industry. ISO 31000 (International Organization for Standardization 2018) defines risk as the effect of uncertainty on objectives. The purpose of research in this context is to establish the scientific basis or understanding of a problem so that mitigation measures or solutions can be developed on a more reliable basis; i.e. research should decrease uncertainty of objectives. Many problems can be identified, but not all have high impact or occur frequently. Risk assessment methods therefore offer a methodology to rank R\&D projects on the basis of their impact. Although not explicitly related to research projects, an example of risk ranking in the mining industry (Ontario Ministry of Labour 2015) identified the rockburst problem as the highest risk of over 260 rated hazards. Rockbursting is highly complex and has been a subject of research for decades, and is a good example of a hazard that requires an integrated approach consisting of sustained long-term basic research in addition to short-term management objectives. No matter how these are allocated, the high ranking of the hazard warrants a sustained allocation of resources. 
Although risk assessment can be adapted to ranking research projects, when allocating resources, there is always a bias towards short-term development work at the expense of long-term basic research. For healthy and sustainable R\&D, it is vital to maintain the pipeline of research spanning the full range from basic to applied investigations. This process can be facilitated by using classification schemes.

\subsection{Classification of research topics}

Classification systems are developed to highlight and quantify the principal components of problems, and are constructed to enable the characteristics of those components to be categorised in order to tap into empirical experience (e.g. rock mass classification), or to facilitate other aspects of decision-making. As outlined in the previous sections, decisions often need to be made about how to manage the solution process for a particular problem or application in the context of what type of research and organisation are best suited to do the work. These decisions are not always easy to make, so the use of classification systems is proposed as one tool that can be used to assist with this process. Their use with various applications of LiDAR imaging is illustrated herein. With LiDAR imaging, the process is dependent on the availability of two principal components - hardware and software. Different applications have different requirements of each in terms of software complexity and hardware resolution or other characteristics. For illustration purposes, it is assumed that universities are best suited to developing or refining basic algorithms of analysis and writing software, and that manufacturers are best suited to developing the hardware. All the applications may be divided into four categories from currently available technology to applications which require more fundamental development of analysis methods and hardware (Figure 1).

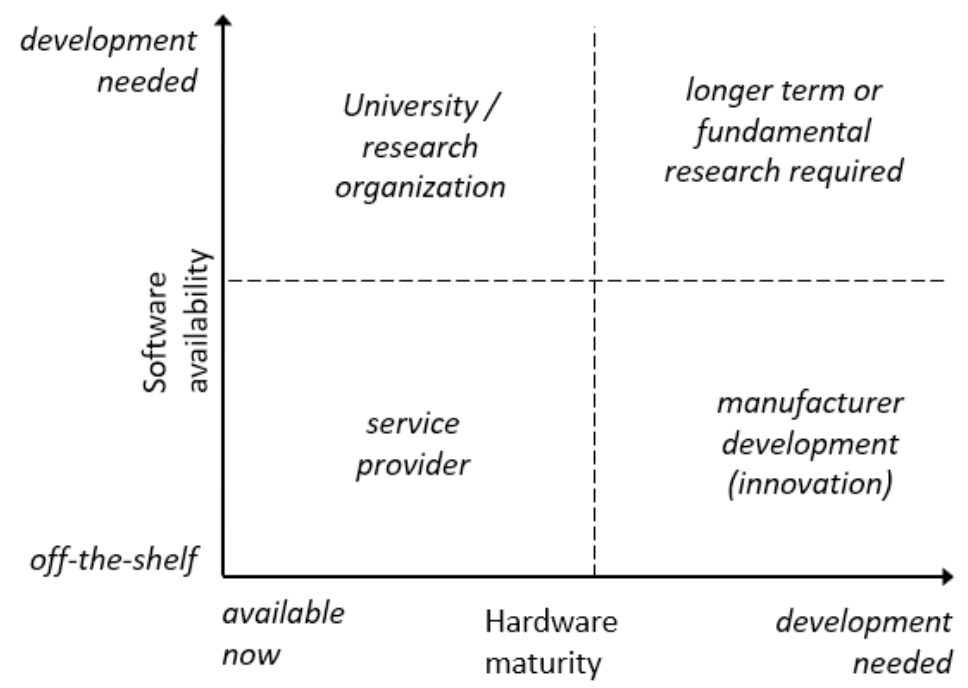

Figure 1 Classification of LiDAR-based applications according to hardware and software status

A selection of imaging tasks may be ranked according to their software and hardware requirements (Figure 2). This system was originally developed to demonstrate what type of project would be best suited for a university graduate student research project, but such a system has wider application in developing strategies and managing a variety of investigations that a mining company might be considering. The example is similar to a conceptual level feasibility study. Should more details be required for planning, further refinements to the categories, such as time requirements, cost, technical difficulty and other parameters could be included and rated using a scoring system. 


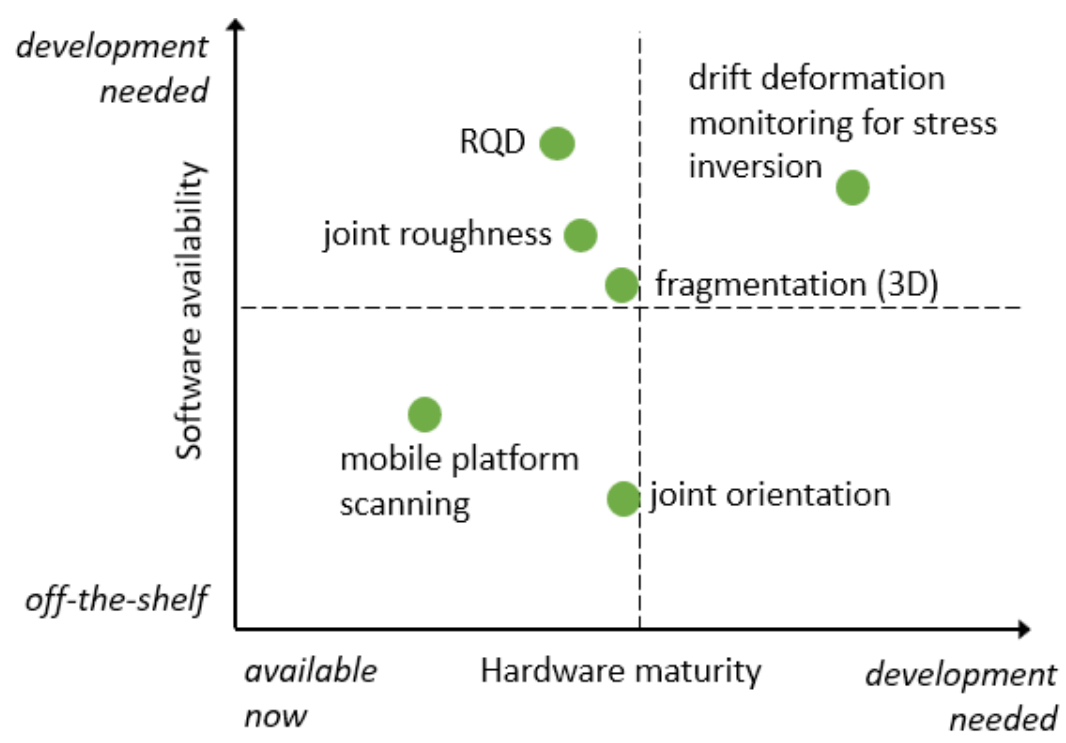

Figure 2 Classification of several mining-related applications of LiDAR

Such systems provide a rational basis for understanding the maturity of a problem, i.e. where on the R\&D spectrum it should be placed and who should work on it, and helps with the allocation of resources of personnel, budget, and time. They may also show connections between themes, i.e. the progression of work required to develop understanding from fundamental investigation to implementation.

\section{$7 \quad$ Research opportunities in cave mining}

Mining spans a large range of sub-disciplines, so no general list of research topics can be complete or representative. However, to illustrate the different forms of research as described in this paper, we outline some important topics (somewhat biased towards geomechanics) relevant to cave mining, spanning the range from fundamental to applied research.

\subsection{Some fundamental problems}

\subsubsection{Rockbursting}

No list of research topics would be complete without mentioning the rockburst problem. A recent study (Ontario Ministry of Labour 2015) listed over 400 mining-related hazards, carried out formal risk assessment of over 260 of these, and identified rockbursting as the top ranked hazard. Since it is a behavioural problem with chaotic characteristics, this problem cannot be 'solved' in the conventional meaning, but progress is being made in both understanding and managing the consequences. This problem requires sustained fundamental research, in collaboration with earth sciences, but there are many mining-specific aspects that will not be addressed by research in the related disciplines alone.

Some issues related to rockburst research include:

- Seismic data remains vastly underutilised.

- This is a technically challenging problem. Skilled practitioners are in short supply with no clear formal training paths available.

- Resources in proportion to the identified risk do not appear to be in place.

A lot of progress has been made in support design, but there are still related inconsistencies in design methodology that need to be resolved. 


\subsubsection{Scale in rock mass behaviour}

Cave mines require significant capital investment in the preparation of new caves, and therefore the consequences of unreliable infrastructure stability analyses could be catastrophic. Experience has shown that for an adequate level of reliability for design purposes, numerical stress analysis models need to be calibrated against similar stress/strength conditions. For new caves or new orebodies in different stress or geological conditions, this is not possible. Experience has also shown that the conventional methodologies for rock mass strength parameter estimation and stress field characterisation are not particularly reliable. Geomechanical classification systems have been developed from data gathering and case histories at small scale compared to the volumes of typical cavities. Understanding behaviour across a wide range of scales remains a major challenge.

\subsubsection{Preconditioning the rock mass for caving}

Preconditioning of a rock mass by either blasting (Catalán et al. 2012) or hydraulic fracturing (He et al. 2016; Pardo \& Rojas 2016) has been used for various tasks including inducement of cave propagation, improved fragmentation, and management of induced seismicity. The effects of preconditioning are known, but their controlling mechanisms are not fully understood. Design of their implementation is therefore still somewhat trial-and-error, but much continues to be learned through this process. More formal planning of research into these techniques could complement and lower the risk associated with the live trials.

\subsection{Intermediate-term research problems}

\subsubsection{Scale in production}

Increasing scale of operations and production rate is associated with improved project economics, but there are also higher risks should there be problems in the long development cycle or geomechanical conditions of caving. Other issues include:

- Prediction of caving and fragmentation in more varied and complex geological conditions.

- Management of cave lines as length does not scale well.

- Estimation of seismic hazard, and design of suitable (preconditioning) mitigation measures.

There is a limited experience base for some of these issues, but a more fundamental understanding is required to reduce the risk of not achieving design performance. Research on these topics should start many years before the design stage of large-scale caving operations.

\subsubsection{Mud rushes}

A chronic operational problem, detection of water in drawpoints can lead to their closure due to the risk of mud rushes. A great deal has been learned about the factors contributing to the formation of mud rushes (Widijanto et al. 2012; Jakubec et al. 2012) and their triggers, allowing safety protocols to be developed. However, research into their formation in the draw column, detection, and risk assessment of mud rush formation, is required to develop and improve the reliability of appropriate mitigation strategies (e.g. drainage, controlled disturbance of the draw column).

\subsubsection{Layouts to avoid production excavation development ahead of cavities}

Exposure of crown pillars to the passage of high abutment stresses during cave line advance, and the resultant damage, is a chronic problem with conventional undercutting. At greater depth, stresses during development are sufficient to cause fracturing and overbreak that can weaken the production structures in advance of their operational life. Modifications to crown pillar design, including removal of orepasses from within production level layouts, can help to alleviate the high stress problem, as can macrosequence design and other details of cave line geometry. Advance undercut held great promise in this regard, but gave rise to 
other geomechanical problems. To preserve production excavation integrity in high-stress conditions, advances in these various factors affecting performance of production level excavations, including methods of modifying the response of the rock mass to high stresses, will produce significant benefits.

\subsection{Innovation}

\subsubsection{Data mining}

Mining is typically data poor, which is why experience is so highly valued. However, in certain specific cases, new technologies are making vast amounts of data available, but methodologies for collecting, analysing, interpreting and making use of this data remain largely undeveloped. Examples of such data sources include induced seismicity, LiDAR, satellite imagery, digital photogrammetry, and continuously monitored equipment. Significant advances in data mining techniques, specifically developed to identify patterns and relationships in vast amounts of data, remain to be applied to these sources of data. This represents an opportunity to develop new empirical relationships, and to provide the foundations of well-documented datasets that can be used further to develop more fundamental understanding, or to calibrate quantitative models.

\subsubsection{Imaging, sensing, Global Positioning System}

New digital instruments are constantly making their way into the mining environment, and others remain to be adapted. However, there are many cases where their application is limited through lack of application or analysis software relevant to mining. Significant opportunities therefore exist in these applications. Sensing, imaging, and positioning technologies will provide the foundational data to form the virtual digital mine, which will be the basis for many other technological applications, including training, digital documentation, and automation.

\subsubsection{Virtual and augmented reality}

The mining industry is in many ways still stuck in a 2D world, but cave mining in particular is complex and 3D. Computer-aided design packages help, but are fundamentally projections of 3D onto 2D. Visualisation of fully 3D environments through virtual and augmented reality is now possible but has not found its way into mining practice. Tools have been developed in other fields, and there is opportunity to build on these to develop new mining-specific applications. The very act of viewing in full 3D can be useful as the brain is a remarkable processor of 3D information. Virtual reality and augmented reality will permit examination and integration of large amounts of data in 3D (plus time, if available), including virtual site visits to problem areas that can be scanned using remote and potentially autonomous mobile (e.g. drones) imaging platforms.

\subsubsection{Integration}

Many problems are complex and increasingly multidisciplinary, or involve collection of diverse types of information and datasets. Synthesis is required, but difficult to define. This will require fundamental thinking of the connections between different aspects of behaviour, factors monitored, etc. Data mining may find correlations, but understanding those correlations is required for most reliable design applications. Availability of new instruments and data combined with developments and software for data mining will greatly facilitate the important task of integrating disparate, but connected, information.

\subsubsection{Autonomous equipment}

Because of wide interest in this area over the last few decades, there is a well-established global R\&D community. With recent developments in computational capacity and sensing technology (particularly low-cost, robust LiDAR and GPS), there has been rapid development in the area of autonomous vehicles. Introduction of such a major disruptive technology into operating mines will require redesign of many existing systems, including mine layouts, monitoring, communication systems, maintenance, training of 
operators and maintenance personnel with new skills. These require considerable development, and are likely the bottleneck, since development of the autonomous equipment is largely external to the mining field.

\section{Conclusion and recommendations}

It is concluded that the nature of much of the mining industry, small to medium size operations with short-term lives (say five to 10 years), are unlikely to have the funds to invest in R\&D, and would be unable to actually benefit from medium to long-term research.

On the other hand, substantial mineral deposits extracted by large-scale caving methods, are likely to have long-term life spans and suffer a number of problems over their long lives. Such operations are thus likely to have the need and the necessary funds to invest in R\&D.

There is a case, therefore, for large-scale caving mining companies to consider the potential for problems over long life mines, and to put in place research programs to resolve potential problems in advance. Given the mobility of technical workforces today and the reach of social media, researching problems with a view to patenting solutions or keeping the advances made secret for competitive advantage is an approach with scant chance of success.

It is important that chronic or systemic problems of the industry be identified and a strategy formulated to solve them.

In a fashion similar to the South African Chamber of Mines set-up in the last century (by the principal mining houses at that time), to study and resolve the rockburst problems of deep level hard rock mines (amongst others), a similar organisation to structure and share research programs for mutual gain is recommended.

\section{References}

Catalán, A, Onederra, I \& Chitombo, G 2012, 'A proposed methodology for evaluation of the preconditioning by blasting at the Cadia East panel cave mine', Proceedings of MassMin 2012, Canadian Institute of Mining, Metallurgy and Petroleum, Westmount.

$\mathrm{He}, \mathrm{Q}$, Suorineni, FT \& Oh, J 2016, 'Review of hydraulic fracturing for preconditioning cave mining', Rock Mechanics and Rock Engineering, vol. 49, pp. 4893-4910.

Hustrulid, W 2006, 'Some thoughts about mining, rock mechanics and butterflies', in P Yale (ed.), Proceedings of the 41st U.S. Rock Mechanics Symposium, vol. 1, American Rock Mechanics Association, Alexandria.

International Organization for Standardization 2018, ISO 31000:2018, Risk Management - Guidelines, International Organization for Standardization, Geneva.

Lewis, M 2016, The Undoing Project: A Friendship That Changed Our Minds, W. W. Norton \& Company, New York City.

Jakubec, J, Clayton, R \& Guest, AR 2012, 'Mudrush risk evaluation', Proceedings of MassMin 2012, Canadian Institute of Mining, Metallurgy and Petroleum, Westmount.

Leach, A 2014, 'Is increasing mining R\&D the only hope for saving a stalling industry?', Mining Technology, viewed 25 July 2018 , https://www.mining-technology.com/features/featureis-increasing-mining-rd-the-only-hope-for-saving-a-stalling-industry4274633/

Mencken, HL 1917, 'The divine afflatus', New York Evening Mail, 16 November 1917.

Minerals Council of Australia 1998, Back from the Brink, viewed 25 July 2018, http://www.mineralscouncil.com.au/file_upload/ files/resources/mtec/backfromthebrink_exec_summ.pdf

Ontario Ministry of Labour 2015, Final Report: Mining Health, Safety and Prevention Review, viewed 25 July 2018 , https://www.labour.gov.on.ca/english/hs/pubs/miningfinal/

Pardo, C \& Rojas, E 2016, 'Selection of exploitation method based on the experience of hydraulic fracture techniques at the EI Teniente mine', Proceedings of MassMin 2016, The Australasian Institute of Mining and Metallurgy, Melbourne.

Widijanto, E, Sunyoto, WS, Wilson, AD, Yudanto, W \& Soebari, L 2012, 'Lessons learned in wet muck management in Ertsberg East Skarn System of PT Freeport Indonesia', Proceedings of MassMin 2012, Canadian Institute of Mining, Metallurgy and Petroleum, Westmount. 
